\title{
A Rule Extraction Method Using Relevance Factor for FMM Neural Networks
}

\author{
Seung Kang Lee ${ }^{+} \cdot$ Jae Hyuk Lee ${ }^{++} \cdot$ Ho Joon Kim ${ }^{+++}$
}

\begin{abstract}
In this paper, we propose a rule extraction method using a modified Fuzzy Min-Max (FMM) neural network. The suggested method supplements the hyperbox definition with a frequency factor of feature values in the learning data set. We have defined a relevance factor between features and pattern classes. The proposed model can solve the ambiguity problem without using the overlapping test process and the contraction process. The hyperbox membership function based on the fuzzy partitions is defined for each dimension of a pattern class. The weight values are trained by the feature range and the frequency of feature values. The excitatory features and the inhibitory features can be classified by the proposed method and they can be used for the rule generation process. From the experiments of sign language recognition, the proposed method is evaluated empirically.
\end{abstract}

Keywords : FMM Neural Network, Rule Extraction, Pattern Recognition

\section{FMM 신경망에서 연관도요소를 이용한 규칙 추출 기법}

\author{
이 승 강 $\cdot$ 이 재 혁 ${ }^{+\dagger} \cdot$ 김 호 준 ${ }^{+\dagger}$
}

\section{요 약}

본 연구에서는 수정된 구조의 FMM 신경망으로부터 패턴 인식을 위한 규칙 추출 방법을 제안한다. 제안된 방법은 학습데이터에서 특징값에 대한 빈도 요소를 반영하는 하이퍼박스 정의를 기반으로 하는데, 이로부터 특징과 패턴클래스 간의 상호 연관도 요소를 정의 하였다. 이는 기 존의 모델에서 사용되는 하이퍼박스 중첩테스트 및 축소(contraction) 기법을 사용하지 않아도 하이퍼박스의 중첩에 의한 분류의 모호성을 해결 할 수 있게 한다. 본 연구에서는 패턴 클래스의 각 차원별로 퍼지 분할을 기반으로 하는 수정된 하이퍼박스 멤버쉽 함수와 이를 사용하는 학습 방법을 제시한다. 제안된 기법으로부터 특정패턴의 분류를 위한 자극성(excitatory) 특징 및 억제성(inhibitory) 특징을 구분하고 이들 정보는 규 칙 생성과정에 적용된다. 수화 인식에 관한 실험에 제안된 방법론을 적용함으로써 제안된 이론의 타당성을 실험적으로 고찰하였다.

키워드 : FMM신경망, 규칙추출, 패턴인식

\section{1. 서 론}

퍼지 최대최소(FMM:Fuzzy Min-Max) 신경망은 하이퍼 박스 기반의 패턴분류 모델이다[1-3]. FMM 신경망 모델은 Simpson[2]에 의해 최초로 제안된 이후에 클러스터링 문제 와 패턴 분류 문제를 위하여 모델의 특성을 일반화한 연구

\footnotetext{
※ 본 연구는 교육과학기술부와 한국연구재단의 지역혁신인력양성사업으로 수행된 연구결과임.

* 이 연구는 한동대학교 교내연구지원사업 제 20120028호에 의한 것임

※ 이 논문은 한국정보처리학회 제 38 회 추계학술발표대회에서 'FMM 신경 망에서 연관도요소를 이용한 규칙 추출 기법'의 제목으로 발표된 논문을 확장한 것임.

† 준 회 원: 한동대학교 정보통신공학과 석사과정(주저자)

†† 준 회 원 : 한동대학교 전산전자공학부 학사과정

†† 종신회원: 한동대학교 전산전자공학부 교수

논문접수: 2012년 12월 24 일

수 정 일 : 1 차 2013년 1 월 18 일

심사완료 : 2013년 1월 18일

* Corresponding Author:Ho Joon Kim(hjkim@handong.edu)
}

가 발표된 바 있으며[1], 최근 Quteishat 등의 연구에서는 FMM 신경망에서 유전자 알고리즘 기반의 규칙 생성 기법 을 제안하였다[3]. 그러나 이들 연구에서 제시한 모델은 학 습데이터에서 특징값의 빈도요소를 효과적으로 반영하지 못 한다는 약점을 갖는다. 다시 말해서 다수의 학습데이터에 의해 학습된 신경망이 극소수의 왜곡된 추가학습 데이터만 으로 급격한 성능저하를 보일 수 있다는 단점을 갖는다. 또 한 하이퍼박스에서 각 차원별로 특징값 범위의 폭이 달라질 수 있음으로 인해 특징의 상대적 연관도를 효과적으로 반영 하지 못한다. 이에 본 연구에서는 학습데이터에서 특징값의 빈도요소를 반영하는 활성화 특성과 학습기법을 제안함으로 써 소수의 데이터에 의한 성능왜곡을 방지할 수 있게 하며, 학습과정에서 하이퍼박스 축소과정을 수행하지 않고도 분류 의 모호성을 극복할 수 있게 한다. 또한 학습된 신경망으로 부터 특징과 패턴클래스간의 연관도요소를 정량화함으로써 
규칙기반 형태의 지식생성을 가능하게 한다.

이전의 연구에서 수정된 FMM 기반의 수화패턴인식 기 법[4]을 제시한바 있는데 본 논문에서는 이에 대한 확장으로 서 패턴인식을 위한 지식추출의 방법론을 소개하고 수화인 식 문제[5]를 대상으로 타당성을 고찰한다.

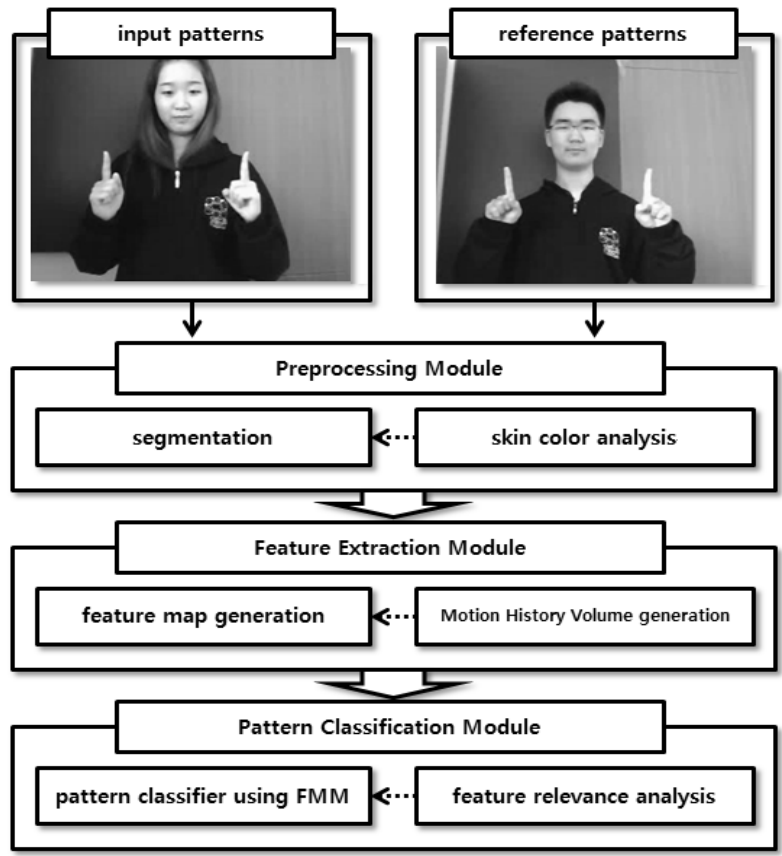

Fig. 1. The structure of the sign language recognition system

수화인식 문제나 제스처인식 등의 문제에서는 시간차원을 포함하는 데이터의 동적 특성을 고려하여야 하고, 시점 (view point)의 변화에 따른 패턴형태의 변화를 고려하여야 하므로 이에 따른 다양한 방법론을 연구해야 한다[4-9]. 본 연구에서 대상으로 하는 시스템은 Fig. 1 과 같은 구조의 다 단계 인식 모델을 전제로 한다. 그림에 보인 바와 같이 총 3 단계의 과정을 수행하는데, 전처리 단계에서는 수화 동영상 패턴으로부터 손동작 등의 인식을 위하여 피부 색상 등을 분석하여 대상영역을 분할하는 과정을 수행한다. 이어서 특 징추출 단계에서는 3 차원 구조의 특징지도를 생성하게 되는 데 이때 $\mathrm{CNN}$ (Convolutional Neural Networks) 모델 기반의 특징추출 기법을 적용한다. 패턴 분류 단계에서는 특징의 연관도 요소를 분석하고 이로부터 패턴 클래스를 분류하게 된다. 이 과정을 위하여 수정된 구조의 $\mathrm{FMM}$ 모델을 사용 하였는데 학습된 신경망으로부터 일련의 규칙기반을 추출할 수 있음을 보인다.

수화인식에서 $\mathrm{CNN}$ 모델[6]을 사용하여 특징을 추출하는 방법을 사용하였는데 이 때 특징의 공간적 위치 및 시간적 변이를 수용할 수 있도록 하기 위하여 3차원 수용영역 (receptive field) 구조로 구현하였다. 동영상으로부터 수화패 턴 데이터의 표현은 모션 히스토리 볼륨(MHV: Motion History Volume) 구조를 사용하였는데 이는 움직임 정보를
시간차원으로 확장 추출함으로써 생성된다. 이로부터 $\mathrm{CNN}$ 모델을 적용하여 3 차원 구조의 특징지도를 생성함으로써 특 징의 수를 현격하게 줄일 수 있을 뿐만 아니라, 특징의 변 이에 강인한 인식기능을 제공할 수 있다. 본 연구에서는 6 가지 수화패턴에 대하여 27 개 특징요소에 대한 상대적 연관 도 요소로부터 제안된 학습방법과 지식 추출방법의 타당성 을 고찰한다.

\section{MHV과 CNN 모델을 사용한 수화 인식}

본 연구에서는 모션 히스토리 볼륨(MHV: Motion History Volume)의 특징으로부터 수화 패턴을 인식하는 문 제를 고려한다. 이는 영상에서 움직임 정보의 시간에 따른 변화를 반영하는 3 차원 볼륨 형태로서, 수화 패턴에 대한 동영상의 각 프레임으로부터 특징영역을 추출하고 이에 대 한 변화를 시간차원에따라 확장함으로써 생성된다. $\mathrm{CNN}$ 모 델은 C-계층(Convolutional Layer)과 S-계층(Subsampling Layer)이 다층구조로 연결된 신경망이다[5]. 상위 계층으로 갈수록 점진적으로 대상영역이 확장되는 형태의 특징지도를 생성하며 이 과정에서 수용영역(receptive field)의 연결 구 조를 통하여 특징점의 위치이동에 강인한 추출기능을 제공 한다. 본 연구에서는 Fig. 2에 보인 바와 같이 수정된 구조 의 $\mathrm{CNN}$ 모델을 적용한다.

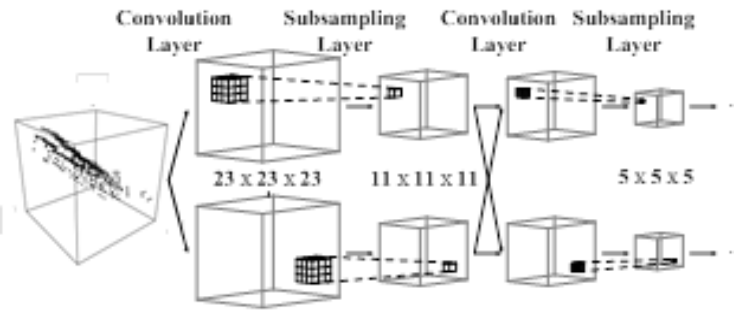

Fig. 2. The extended CNN model for feature extraction

그림에 보인 바와 같이 입력데이터 및 특징지도를 3차원 구조로 표현하게 되는데 이는 기존의 $\mathrm{CNN}$ 모델에서 수용영 역(receptive field)을 3차원으로 확장한 형태이며 계층구조 에 따라 점진적으로 확장된 영역을 반영하여 특징수를 줄여 나갈 수 있게 한다. 다시 말해서 최종적으로 생성되는 볼륨 형태는 Fig. 3과 같이 특징영역에 대하여 상하, 좌우 및 시 간에 대한 변화를 보이게 된다.

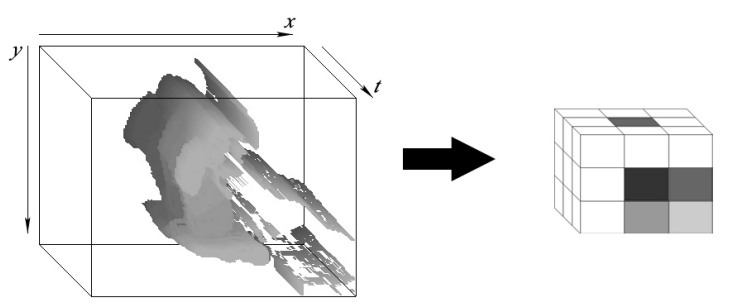

Fig. 3. An example of feature map 
추출되는 특징지도의 형태는 3 차원 수용영역 구조를 따라 점진적으로 축소되어, 최초데이터를 $(21 \times 21 \times 21)$ 의 크기 로 고려하고 최종 특징지도를 $(3 \times 3 \times 3)$ 으로 산출한다면 약 450 분의 1 로 데이터양을 줄일 수 있게 된다. 특징지도의 생성과정은 다음과 같다. 입력 신호에서 생성된 $\mathrm{MHV}$ 에서 다음단계의 계층으로 샘플링 되고 중첩된 구조로 인접영역 을 조사하게 된다. 다단계로 추출된 데이터는 대상영역이 점진적으로 확대되고 특징수가 감소하게 된다. 계층 $l$ 에서 의 $i$ 번째 위치에 대한 특징지도 값 $C_{i}^{l}$ 은 다음 식과 같이 표현된다.

$$
C_{i}^{l}=g\left(N_{i, l} \bigcirc W_{l}+B_{i, l}\right)
$$

식에서 $N_{i, l}$ 은 계층 $l$ 에서 $i$ 번째 위치의 3 차원 인접영역 (neighborhood)을 의미하며, $W_{l}$ 은 해당 계층에서 추출된 특 징의 형태에 따라 결정되는 가중치이다. 또한 $B_{i, l}$ 은 바이어 스 값을 의미하며 연산 $\bigcirc$ 는 3 차원 convolution 연산을 의 미한다. 함수 $g$ 는 $\tanh (x)$ 형태의 시그모이드 함수이다. 반 면 S-계층에서는 동일한 수용 영역 구조를 따라 다운 샘플 링을 하게 된다. 이 때 하위계층에서 감시되는 영역 내에서 특징의 존재여부를 반영하므로, 3 차원 공간상에서 특징점의 위치 변화를 일정량 허용하게 된다. 반면 $\mathrm{C}$-계층과 연결된 가중치는 3 차원 수용영역 구조로부터 중앙노드를 중심으로 3 차원 가우시안 분포를 갖도록 설정한다. 다시 말해서 하위 계층에서 특징점의 3 차원 공간상의 변이, 즉 공간적 및 시 간적 변이를 수용하는 기능을 지원하게 된다.

\section{3. 수정된 FMM 신경망과 규칙 추출 기법}

FMM 신경망을 사용하여 패턴 분류를 위한 규칙을 추출 하는 방법이 최근의 연구에서 제안된 바 있다[3]. 이 연구에 서는 오픈 하이퍼박스를 고려하여 가능한 모든 조합의 하이 퍼박스를 가상하여 스트링을 정의하고 유전자 알고리즘 $(\mathrm{GA}$ : Genetic Algorithm)에 의한 최적화기법을 사용하여 특징과 패턴클래스 간의 'If-then rule'의 집합을 생성하였다. 그런 데 이 연구에서는 유전자로 고려하는 특징의 조합이 매우 방대하게 증가하는 문제와 학습데이터의 빈도요소를 고려하 지 못한다는 취약점을 갖는다. 이에 본 연구에서는 수정된 특성을 갖는 $\mathrm{FMM}$ 모델을 정의하고, 이로부터 특징과 패턴 클래스간의 규칙을 생성하는 방법론을 제안한다.

임의의 하이퍼박스 $j$ 의 멤버쉽함수는 식 (2)과 같이 정의 된다.

$$
B_{j}\left(A_{h}\right)=\frac{1}{Z} \sum_{i=1}^{n} w_{j i} \cdot f\left(a_{h i}, I_{j i}\right)
$$

식에서 $A_{h}=\left(a_{h 1}, a_{h 2}, \cdots, a_{h n}\right) \in I^{n}$ 는 $h$ 번째 입력패 턴으로 총 $\mathrm{n}$ 개의 특징값들로 이루어진다. 또한 $I_{j i}$ 는 하이퍼
박스 $j$ 에서 $i$ 번째 차원에 대한 특징구간을 의미한다. $Z$ 는 정규화를 위한 상수이며, $w_{j i}$ 는 $i$ 번째 특징과 $j$ 번째 하이퍼 박스간의 가중치 요소이다. 함수 $f(\cdot)$ 는 식 (3)와 같이 결 정된다.

$$
f\left(a_{h i}, I_{j i}\right)= \begin{cases}1.0 & \text { if } I_{j i}^{L}<a_{h i}<I_{j i}^{U} \\ 1.0+\gamma\left(a_{h i}-I_{j i}^{L}\right) & \text { if } a_{h i}<I_{j i}^{L} \\ 1.0-\gamma\left(a_{h i}-I_{j i}^{U}\right) & \text { if } a_{h i}>I_{j i}^{U}\end{cases}
$$

학습과정에서 하이퍼박스 확장(expansion)은 기존의 방법 과 유사하게 이루어지는데, 부수적으로 가중치 값이 변경된 다. 가중치 값은 해당특징 범위의 폭과 학습데이터에서 발 생한 특징의 빈도에 의해 학습되는데 그 규칙은 식 (4)과 같다.

$$
w_{j i}^{\text {new }}=\left\{\begin{array}{l}
w_{j i}^{\text {old }}+\lambda \quad \text { if }\left(\left|I_{j i}\right|^{\text {old }}=\left|I_{j i}\right|^{\text {new }}\right) \\
\frac{w_{j i}^{\text {old }} \cdot\left|I_{j i}\right|^{\text {old }}}{\left|I_{j i}\right|^{\text {new }}} \text { otherwise }
\end{array}\right.
$$

다시 말해서 특징구간의 변화가 없는 상태에서 반복학습 이 되면 가중치가 증가하고, 폭이 증가하면 그 크기에 반비 례하여 가중치가 감소하게 된다. 이러한 가중치 요소는 학습 데이터에서 특징값의 빈도(frequency)요소를 반영하여 서로 다른 값으로 결정된다. 그로 인하여 특정차원에서 동일 특징 점에 대해서도 서로 다른 값으로 하이퍼박스 멤버쉽에 반영 되므로 하이퍼박스의 중첩(overlapping)의 판별이나 축소 (contraction)과정 없이도 분류의 모호함을 해결할 수 있다.

이와 같은 방법으로 학습된 $\mathrm{FMM}$ 신경망으로부터 특징 과 패턴클래스간의 상호 연관도 요소(relevance factor)를 산 출할 수 있다. 본 연구에서는 특징구간 $I$ 와 패턴클래스 $k$ 간 의 연관도값 $R F(\cdot)$ 를 식(5)와 같이 정의 한다.

$$
R F\left(I_{j i}, k\right)=\left(\frac{1}{N_{k}} \sum_{B_{j} \in C_{k}} w_{j i}-\frac{1}{N_{B}-N_{k}} \sum_{B_{j} \notin C_{k}} w_{j i}\right) / \sum_{B_{j} \in C_{k}} w_{j i}
$$

식에서 $C_{k}$ 는 클래스 $k$ 에 속하는 하이퍼박스의 집합이며, $N_{B}$ 및 $N_{k}$ 는 각각 전체 하이퍼박스의 개수 및 클래스 $k$ 에 속하는 하이퍼박스의 개수를 의미한다.

특징 구간 $I$ 의 표현은 Fig. 4 와 같이 5 가지 종류의 퍼 지 분할로 구분하고 연속된 분할을 포함하여 구간을 결정 한다.

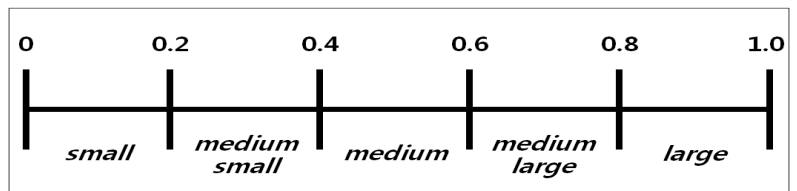

Fig. 4. The fuzzy partition defined for each dimension of a pattern class 
식 (5)의 연관도 요소로부터 패턴클래스 $k$ 에 대한 자극 성 (excitatory) 특징 및 억제성(inhibitory) 특징을 분류할 수 있고 이로부터 규칙의 집합을 생성할 수 있다. 규칙의 신뢰도 요소(CF: Confidence Factor)는 $\mathrm{RF}$ 의 절대값으로 부 터 산출된다.

\section{4. 실험 결과}

첫 번째 실험은 하이퍼박스 중첩영역에 대한 패턴 분류 성능 평가에 대한 실험이다. 잘 알려진 Fisher의 아이리스 표준 데이터로부터 총 10 개 패턴과 2 개의 특징만으로 단순 화 시킨 실험에서 하이퍼박스 중첩영역의 영향을 평가 한 내용이다. Fig. 5의 (a)에서 X표시 된 부분은 중첩영역에 나타난 패턴 클래스 $\mathrm{B}$ 에 속한 패턴으로 기존 모델의 문제 점을 보이는 예이다. 다시 말해서 그림에서 (b)의 형태는 하이퍼박스 중첩영역 발생한 형태로 이러한 경우의 모호성 을 해결하기 위하여 축소(contraction)과정을 수행하여 (a) 의 형태로 조정되게 되는데 이러한 중첩영역에서 분류 오 류가 발생함으로서 성능이 저하되게 된다. 반면 제안된 모 델에서 가중치 요소는 서로 다른 값으로 반영됨으로써 그 림에서 $(\mathrm{d})$ 에 보인바와 같이 중첩영역에서도 각 하이퍼박 스에 대한 소속 함수 값이 서로 다른 값으로 나타날 수 있 음을 보이고 있다.
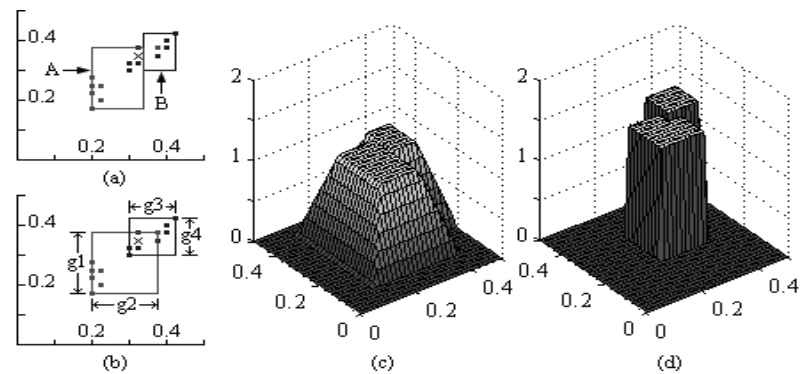

Fig. 5. Comparison of the two different decision makings inside the hyperbox region

두 번째 실험은 수화패턴에 대한 인식 실험이다. 본 연구 에서는 기본적인 수화패턴으로, '인사(greeting)', '만남 (meet)', '이별(depart)', '반가움(glad)', '아쉬움(regret)'및 '감 사(thank you'’등 총 6가지 패턴을 대상으로 고려하였다. Fig. 6는 본 연구에서 대상으로 한 수화인식 시스템의 사용 자 인터페이스의 예를 보인 것이다.

Fig. 7은 특징추출을 위하여 수화패턴을 $\mathrm{MHV}$ 의 형태로 표현한 예이다.

이로부터 $\mathrm{CNN}$ 모델에 의하여 3 차원 볼륨형태의 특징지 도를 생성하게 되는데, $(3 \times 3 \times 3)$ 크기 구조의 볼륨에서 각 단위 데이터는 $\{$ 상, 중, 하 $\}$ 의 의미와 $\{$ 좌, 중, 우 $\}$ 의 의미 를 갖는 공간적 위치와 \{초반, 중반, 종반\}의 의미를 갖는 시간적 위치를 표현하게 된다. Table 1 은 학습패턴에 대하 여 특징값을 추출한 결과의 예이다.

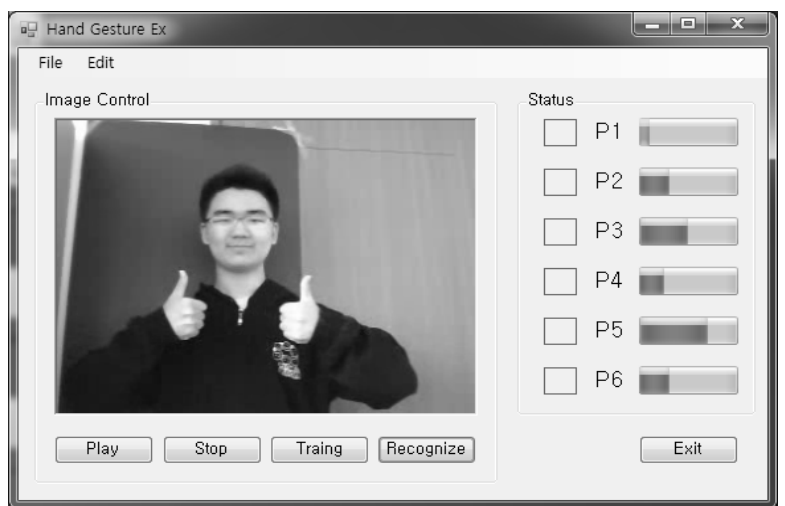

Fig. 6. An example of the user interface of sign language recognition system
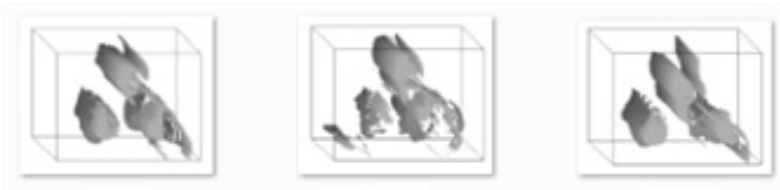

(a)
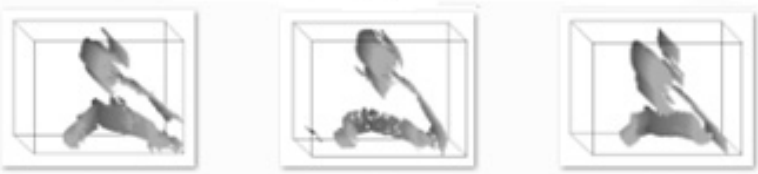

(b)

(a) greeting, (b) depart

Fig. 7. Examples of Motion History Volumes

Table 1. Examples of feature sets of sign language patterns

\begin{tabular}{c|c|c|c|c|c|c}
\hline Pattern & P1 & P2 & P3 & P4 & P5 & P6 \\
\hline Meaning & greeting & meet & depart & glad & regret & $\begin{array}{c}\text { thank } \\
\text { you }\end{array}$ \\
\hline a1 & 0 & 0 & 0 & 0.251 & 0.203 & 0.268 \\
\hline $\mathrm{a} 2$ & 0 & 0.001 & 0.003 & 0.016 & 0.134 & 0.279 \\
\hline $\mathrm{a} 3$ & 0 & 0 & 0.004 & 0.029 & 0 & 0 \\
\hline$\cdots$ & $\cdots$ & $\cdots$ & $\cdots$ & $\cdots$ & $\cdots$ & $\cdots$ \\
\hline $\mathrm{a} 25$ & 0.014 & 0 & 0.246 & 0.141 & 0 & 0 \\
\hline $\mathrm{a} 26$ & 0.036 & 0.001 & 0.290 & 0.356 & 0.262 & 0.354 \\
\hline $\mathrm{a} 27$ & 0.154 & 0.001 & 0.149 & 0.333 & 0.156 & 0 \\
\hline
\end{tabular}

본 논문의 실험은 특징의 연관도 요소를 산출하고 이로부 터 규칙기반 형태의 지식을 추출하는 것이다. 따라서 다양 한 특징의 종류를 사용하지 않고 수화패턴에서 손 특징에 대한 움직임 정보만을 대상으로 하였다. 학습된 신경망으로 부터 추출한 연관도 요소의 분석결과의 예를 Table 2에 보 였으며, 이로부터 각 수화패턴에 대하여 자극성 특징 (excitatory features)과 억제성 특징(inhibitory features)을 구분한 결과를 Table 3 에 보였다. 다시 말해서 이 결과로부 터 다음과 같은 규칙기반을 추출할 수 있음을 의미한다. 
Table 2. Relevance factors between features and pattern classes

\begin{tabular}{c|c|c|c}
\hline Feature & Meaning & Pattern & Relevance Factor \\
\hline a1 & Left-upper-early & P1 & 0.250 \\
\hline a5 & Center-middle-early & P3 & -0.493 \\
\hline a12 & Right-upper-middle & P5 & 0.000 \\
\hline a15 & Right-middle-middle & P6 & 0.454 \\
\hline a16 & Left-lower-middle & P6 & -0.137 \\
\hline a22 & Left-middle-latter & P4 & 0.008 \\
\hline a24 & Right-middle-latter & P2 & -1.022 \\
\hline
\end{tabular}

Table 3. Knowledge extraction results on the sign language recognition

\begin{tabular}{c|c|c|c}
\hline Feature & Pattern & Type & RF value \\
\hline a1 & P1 & excitatory & 0.250 \\
\hline a5 & P3 & inhibitory & -0.493 \\
\hline a15 & P6 & excitatory & 0.454 \\
\hline a16 & P6 & inhibitory & -0.137 \\
\hline a22 & P4 & excitatory & 0.008 \\
\hline a24 & P2 & inhibitory & -1.022 \\
\hline
\end{tabular}

지식표현의 예는 다음과 같다.

if $\left(a_{1}\right)$ then 'greeting' with $(c f=0.250)$

if $\left(a_{15}\right)$ then 'thank you' with $(c f=0.454)$

if $\left(a_{5}\right)$ then 'not depart' with $(c f=0.493)$

if $\left(a_{24}\right)$ then 'not meet' with $(c f=1.022)$

\section{5. 결 론}

본 연구에서 제시한 수정된 구조의 $\mathrm{FMM}$ 신경망 모델은 특징과 패턴 클래스간의 상호 연관도 요소를 산출함으로써 기존의 모델에서 하이퍼박스 중첩으로 발생하는 분류 성능 의 저하 현상을 개선하였다. 또한 패턴 인식 문제에서 if-then 규칙 형태의 지식 표현을 생성할 수 있음을 보였다. 본 연구에서 대상으로 한 수화인식 시스템에서 고려하는 특 징표현의 종류는 모션 히스토리 볼륨, 모션에너지 및 동영 상에서의 손의 형태 표현 등이 있다. 본 논문에서는 특징과 패턴클래스간의 상호 연관도 요소의 산출에 중점을 두어 단 순화된 형태의 특징 집합만을 고려하였다. 실용적 성능을 위해서는 대상 수화패턴의 종류를 확장 고려하여 손동작 및 얼굴에 대한 위치정보와 움직임 정보에 관하여 보다 세밀한 특징표현을 고려하여야 한다. 본 연구의 장점으로는 특징표 현과 패턴간의 관계에 대한 전문가의 지식표현으로부터 신 경망의 초기가중치 설정이 가능하고, 실제 데이터를 사용한 학습과정을 통하여 초기 지식이 점진적으로 정련 (refinement)될 수 있다는 점이다. 또한 추출된 지식표현은
학습패턴의 미비점을 파악할 수 있게 하여 추가 학습데이터 의 구성과 학습데이터의 오류에 대한 보완을 보다 효과적으 로 할 수 있게 한다. 향후 연구에서는 보다 다양한 형태의 수화패턴을 대상으로 적용함으로써 제시된 이론을 일반화 하는 연구가 필요한 것으로 판단된다.

\section{참 고 문 헌}

[1] B. Gabrys and A. Bargiela, "General fuzzy min-max neural network for clustering and classification," IEEE Transaction on Neural Networks, Vol.11, No.3, pp.769-783, 2000.

[2] P. K. Simpson, "Fuzzy min-max neural network - Part1 : Classification," IEEE Transaction on Neural Network, Vol.3, No.5, pp.776-786, 1992.

[3] A. Quteishat, C. P. Lim, and K. S. Tan, "A modified fuzzy min-max neural network with a genetic algorithm-based rule extractor," IEEE Transaction on System, Man, and Cybernetics-Part A: System and Humans, Vol.40, No.3, pp.641-650, 2010.

[4] H. J. Kim, "Two-Stage Neural Networks for Sign Language Pattern Recognition", Journal of Korean Institute of Intelligent Systems, Vol.22, No.3, pp.319-327, 2012.

[5] M. M. Zaki and S. I. Shaheen, "Sign language recognition using a combination of new vision based features," Pattern Recognition Letters, Vol.32, No.4, pp.572-577, 2011.

[6] C. Garcia and M. Delakis "Convolutional face finder: A neural architecture for fast and robust face detection," IEEE Transactions on Pattern Analysis and Machine Intelligence, Vol.26, No.11, pp.1408-1423, 2004.

[7] R. Yang and S. Sarkar, "Coupled grouping and matching for sign and gesture recognition," Computer Vision and Image Understanding, Vol.113, No.6, pp.663-681, 2009.

[8] D. Weinland, R. Ronfard and E. Boyer, "Free viewpoint action recognition using motion history volumes," Computer Vision and Image Understanding, Vol.104, pp.249-257, 2006.

[9] A. Yilmaz and M. Shah, "Actions sketch: A novel action representation," IEEE Computer Society Conference on Computer Vision and Pattern Recognition, 2005, pp.984-989.

[10] R. A. Fisher "The use of multiple measurements in taxonomic problems," Annals of Eugenics, Vol.7, No.2, pp.179-188, 1936.

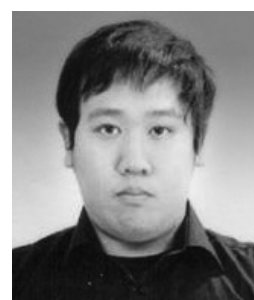

\section{이 승 강}

e-mail :1sk0704@gmail.com

2011년 한동대학교 전산전자공학부

2012년 현 재 한동대학교 정보통신

공학과 석사과정

관심분야: 영상처리, 패턴인식, 컴퓨터비전 


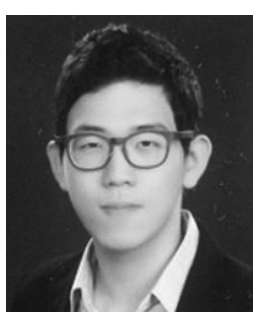

\section{이 재 혁}

e-mail : jh19105@gmail.com

2010년 현 재 한동대학교 전산전자 공학부 학사과정

관심분야: 인공지능, 컴퓨터 보안

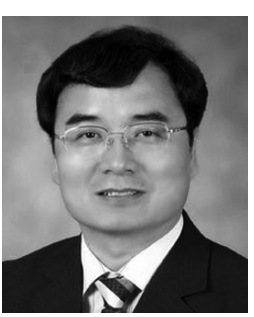

김 호 준

e-mail : hjkim@handong.edu 1987년 경북대학교 전자공학과(학사) 1995년 KAIST 전산학과(공학박사) 1987년 1991년 한국원자력연구소 연구원 2003년, 2010년 미국 캘리포니아주립대 방문교수

1996년 현 재 한동대학교 전산전자공학부 교수 관심분야: 컴퓨터비전, 영상처리, 패턴인식 